\title{
76-Space Analysis of Grey Matter Diffusivity: Methods and Applications
}

\author{
Tianming Liu ${ }^{1,2}$, Geoffrey Young ${ }^{1,2}$, Ling Huang ${ }^{1,2}$, Nan-Kuei Chen ${ }^{2}$, \\ and Stephen TC Wong ${ }^{1,2}$ \\ ${ }^{1}$ Center for Bioinformatics, Harvard Center for Neurodegeneration and Repair, \\ Harvard Medical School \\ ${ }^{2}$ Department of Radiology, Brigham and Women's Hospital
}

\begin{abstract}
Diffusion Weighted Imaging (DWI) and Diffusion Tensor Imaging (DTI) are widely used in the study and diagnosis of neurological diseases involving the White Matter (WM). However, many neurological and neurodegenerative diseases (e.g., Alzheimer's disease and Creutzfeldt-Jakob disease) are generally considered to involve the Grey Matter (GM). Investigation of GM diffusivity of normal aging and pathological brains has both scientific significance and clinical applications. Most of previous research reports on quantification of GM diffusivity were based on the manually labeled Region of Interests (ROI) analysis of specific neuroanatomic regions. The well-known drawbacks of ROI analysis include inter-rater variations, irreproducible results, tediousness, and requirement of a priori definition of interested regions. In this paper, we present a new framework of automated 76-space analysis of GM diffusivity using DWI/DTI. The framework will be evaluated using clinical data, and applied for study of normal brain, Creutzfeldt-Jakob disease and Schizophrenia.
\end{abstract}

\section{Introduction}

DWI/DTI has been widely used to study the WM abnormality associated with disease progression [1], as DTI yields quantitative measures reflecting the integrity of WM fiber tracts, by taking advantage of the intrinsic directionality of water diffusion. However, many neurological diseases, including Alzheimer's disease, are considered to involve the GM $[2,5]$. The diffusivity of GM is widely believed to be able to reflect the neuropathological changes in the neurological and neurodegenerative diseases [2, 5], and can be quantified by the apparent diffusion coefficient (ADC), which provides information on the degree of restriction of water molecules.

To date, applications of DWI/DTI in quantification of GM diffusivity have been done via manual ROI analysis. However, ROI analysis has several notable drawbacks, including inter-rater variations, irreproducible results, tediousness, and requirement of a priori definition of interested regions. In this paper, we present a computational framework of automated 76-space analysis of GM diffusivity. The framework has been evaluated against clinical data, and has been applied to study the GM diffusivity of normal brains, as well as the GM diffusivity abnormalities occurring in Creutzfeldt-Jakob disease and schizophrenia. 


\section{Methods}

\subsection{Overview}

The computational framework of 76-space analysis of GM diffusivity is composed of seven steps, as summarized in Figure 1. The first two steps automatically segment the SPGR brain image into CSF/GM/WM tissues [3], and the GM map is further parcellated into 76 fine-detailed neuroanatomic regions (called 76-space) using the high-dimensional hybrid registration method [3]. The third step performs pre-processing in the DWI/DTI space, e.g., eddy current correction, ADC/FA (Fractional Anisotropy) image generation, reslicing, and co-registration of the B0/ADC/FA images with SPGR image. However, due to potential problems such as EPI geometric distortion, partial volume effect, image reslicing errors, and inaccuracy of co-registration algorithm, the warped B0/ADC/FA images would not be in exact anatomic correspondences with SPGR image. The inaccurate alignment of ADC/FA images with SPGR image prevents us from applying the GM parcellation in the SPGR space directly to the ADC/FA images, as the GM in SPGR space may correspond to heterogeneous tissues e.g., CSF and WM, in the DWI/DTI space. To overcome this problem, Step 4 and 5 segments the brain into CSF/GM/WM tissues in the DWI/DTI space by utilizing the tissue contrasts existing in the ADC image and FA image. Afterwards, Step 6 combines the tissue segmentation results from both SPGR space and DWI/DTI space, and takes the union of the GM maps in both spaces. This AND operation of GM maps results in a GM-Union map, which is classified as GM by both SPGR image segmentation and DWI/DTI image segmentation. Finally, we perform the automated 76-space analysis of GM diffusivity, represented by Step 7 in Figure 1.

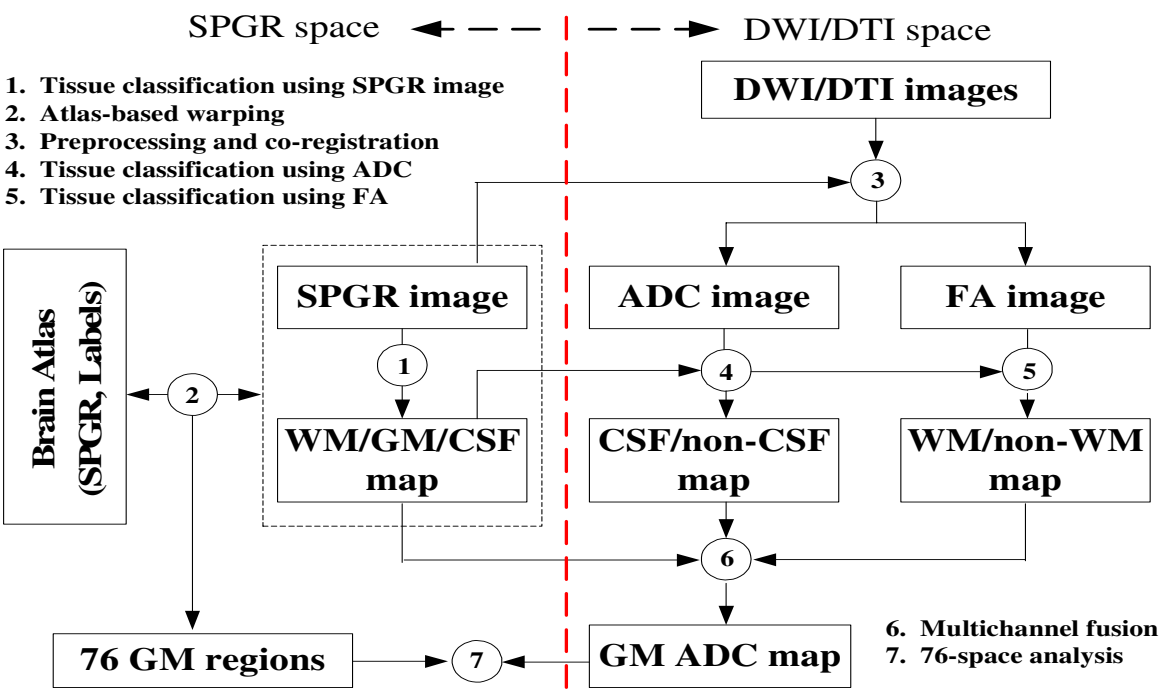

Fig. 1. Illustration of the computational framework of 76-space analysis 


\subsection{GM Parcellation in SPGR Space}

We employ a high-dimensional atlas-based warping method [3] to automatically segment the subject brain SPGR image into 76 neuroanatomic structures. Meanwhile, we apply tissue segmentation on the subject SPGR image, and use the resulted GM map to mask the automatically labeled SPGR image, generating the labeled GM map. Finally, we remove other non-GM tissues by looking at the GM neuroanatomy table, and obtained the $76 \mathrm{GM}$ structures for the following step of 76-space analysis.

\subsection{Tissue Classification in DWI/DTI Space}

\subsubsection{Pre-processing}

For the DWI/DTI data, firstly, we perform the eddy current correction by using the Oxford FSL FDT tools. We calculate the diffusion tensor, and generate the ADC and fractional anisotropy (FA) images using our in-house built tools. Then, we co-register the B0 (no diffusion gradient) image, ADC image and FA image with the SPGR structural image using the linear multimodality registration method of Oxford FSL Flirt. To obtain better co-registration, we further use a non-linear registration method, UCLA-AIR, to produce a more accurate co-alignment between ADC and FA images and SPGR structural image.

\subsubsection{Motivation}

Although the Oxford FSL Flirt and UCLA-AIR algorithms can produce reasonably good co-registration results, a couple of resources still render the possibility that GM regions in SPGR space may include heterogeneous tissues (e.g., CSF and WM) in the DWI/DTI space, and measurements of GM diffusivity may fail to reveal real changes occurring in the GM tissue, if we directly apply the GM segmentation in the SPGR space into the DWI/DTI space. Given that the GM is a laminar with thickness of about $3.0 \mathrm{~mm}$, and the ADC values of the CSF are more than twice as high as the GM values, small errors in co-registration may lead to significant deviation of the measured ADC value in the GM region.

There are multiple reasons causing the problem of heterogeneous tissues. First, there could be geometrical distortions in EPI-generated diffusion tensor images, due to the rapid sampling of the gradient echo train. Second, there is the partial volume effect in MRI. Thirdly, we usually need to reslice the DWI/ADC/FA images, and this reslicing and interpolation process would inevitably generate certain inaccuracies. Fourthly, the accuracy of co-registration methods could be limited. Our solution is to remove the heterogeneous tissues in the GM ADC map by utilizing tissue contrasts existing in the $\mathrm{ADC}$ and FA images. The ADC values in the CSF are more than twice as high as the GM and WM values, because water diffusion in CSF is much less restricted than that in the GM and WM tissues. Therefore, it is straight-forward to use the ADC image to segment CSF from non-CSF tissues. Meanwhile, the FA image can be used to separate WM from non-WM tissues, since highly directional white matter structures have much larger fractional anisotropy values. Importantly, the ADC image and FA image are intrinsically in the same DWI/DTI space, and we can combine the results of CSF/non-CSF and WM/non-WM segmentation results into a complete CSF/GM/WM 
segmentation map in the DWI/DTI space, which is independent of the SPGR space. Then, after taking the union of the GM map in SPGR space and the one in DWI/DTI space, we can substantially remove heterogeneous tissues on the GM ADC map.

\subsubsection{Tissue Classification}

2.3.3.1 HMRF-EM Algorithm. Other than segmenting brain tissues into three classes as in structural MR images, we classify brain tissues into two classes: CSF and non-CSF in ADC images, and WM and non-WM in FA images. We apply a Hidden Markov Random Field (HMRF) model and the Expectation-Maximization (EM) algorithm for the two-class tissue segmentation, which is akin to that in [4].

\subsubsection{Initialization. As both EM model fitting and MRF ICM labeling converge} locally [4], the selection of the initial parameter set is thus very important. In the literature, automated selection of initial centroids methods using k-means classification is widely used for the initial estimation [4]. However, applying k-means method to ADC images for automated selection of initial centroids might be problematic. Figure 2 shows the distribution of the ADC values in the whole brain, in which there are no clear boundaries between different tissues. The k-means method might fail to select the desired initial centroids, rendering difficulty for the MRF ICM labeling and EM model fitting to converge desirably. This may result in the HMRF-EM algorithm being trapped in a local minimum. Recall that we already have the tissue segmentation using SPGR image. We assume that the volume percentage of each tissue (CSF, GM, and WM) segmented in SPGR space should be the same, or close to, that obtained in DWI/DTI space. Hence, the volume percentage of each tissue class obtained in SPGR space can be used as a prior knowledge to guide the automated selection of initial centroids for HMRF-EM segmentation of the ADC image, as illustrated in Figure 2. In Figure 2, we know that the WM and GM occupy $82.4 \%$ of the whole brain volume. This percentage is used to set the ADC threshold, below which are the GM and WM, and their volume sum is the same as $82.4 \%$, as shown in Figure 2. In a similar way, the volume percentage of CSF and GM obtained in SPGR segmentation can be used to set the FA threshold.

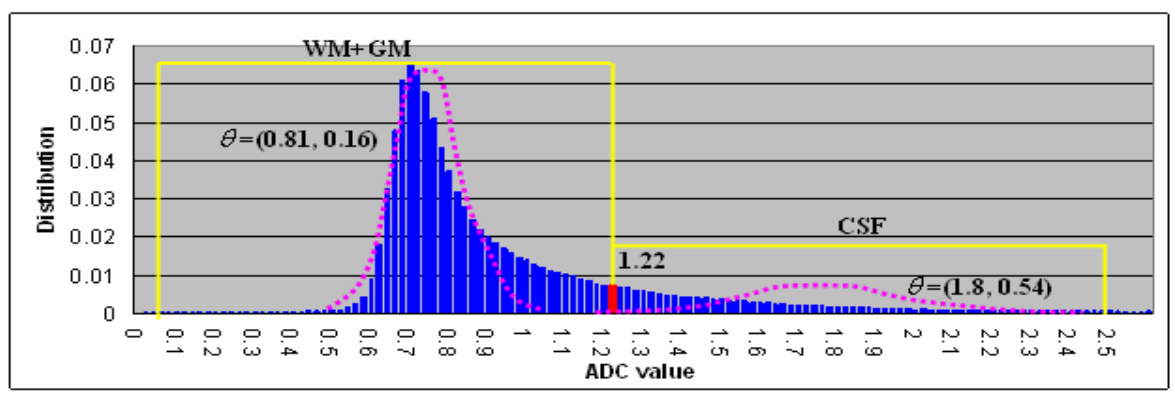

Fig. 2. Initialization for HMRF-EM segmentation of ADC image. ADC scale is $10^{-3} \mathrm{~mm}^{2} / \mathrm{sec}$. The threshold is set such that the total volume percentage below it is exactly the same as 0.824 . The estimated parameters and fitted Gaussian models are overlaid. 


\subsection{Multi-channel Fusion}

The SPGR channel has complete segmentation of CSF, GM and WM (Figure 3b), while the ADC channel has segmentation of CSF and non-CSF (Figure 3f), and the FA channel has segmentation of WM and non-WM (Figure 3j). The aim of multi-channel fusion is to take the union of the SPGR GM map (Figure 3c), the ADC non-CSF map (Figure 3g), and the FA non-WM map (Figure 3k), by performing an AND-like operation, and generating a GM-Union map, as shown in Figure 3o. The AND-like operation ensures that the GM-Union map is the consensus of all three channels. To demonstrate that multi-channel fusion greatly removes heterogeneous tissues, Figure $3 d$ shows the overlap of SPGR GM map (Figure 3c) and ADC CSF map (Figure 3h). Clearly, there are large overlapping areas between CSF tissue in DWI/DTI space and GM tissue in SPGR space. Likewise, Figure 3p shows the overlap of the SPGR GM map (Figure 3c) and the FA WM map (Figure 31). There are also large overlapping areas of WM tissue in the DWI/DTI space and GM tissue in the SPGR space. By using

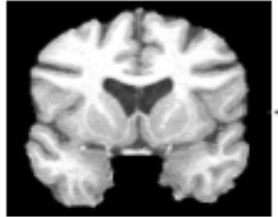

(a) SP GR image

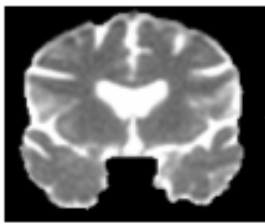

(e) $\mathrm{ADC}$ inage

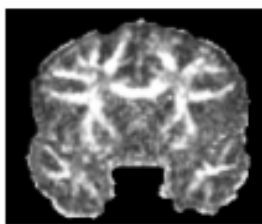

(i) FA image

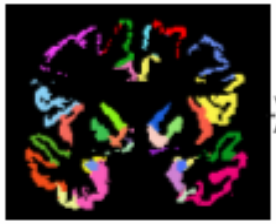

(m) GM 76-space

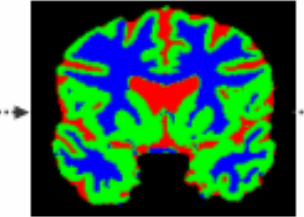

(b) tissue maps

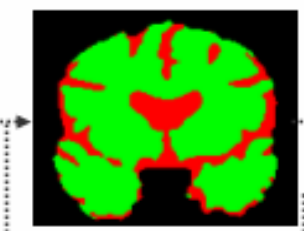

(f) tissue maps

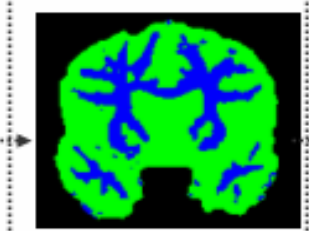

(j) tissue maps

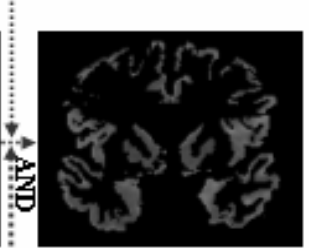

(ii) Gid $\mathrm{ADC}$ map

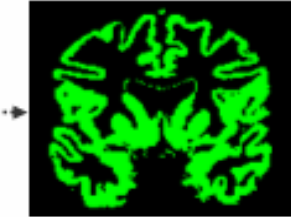

(c) GM

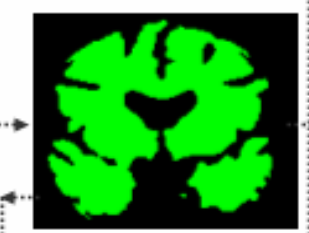

(g) Hon-CSF

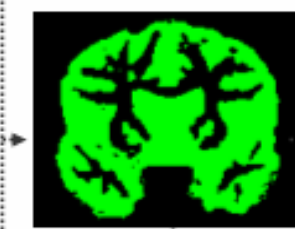

(k) Non-WMI: $\rightarrow$ AND

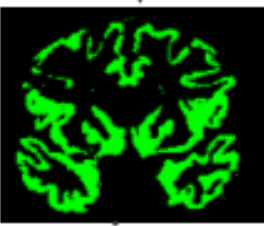

(o) Gulitition

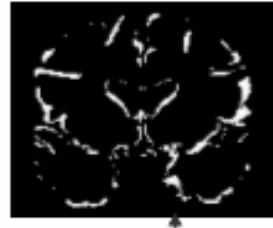

(d) Union of (c) ind (h)

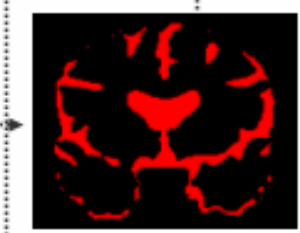

(h) CSF
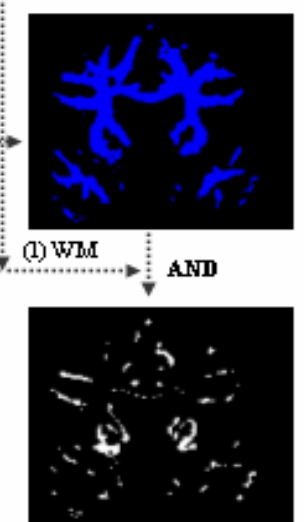

(p) Union of (c) and (1)

Fig. 3. Multi-channel data fusion. The "AND" means taking the union of two maps 
the method in Subsection 2.2, we automatically segment the GM into 76 spaces, as shown in Figure $3 \mathrm{~m}$. Then, we combine the GM-Union map (Figure 3o), the labeled GM map (Figure 3m), and the original ADC map (Figure 3e) together, and we have the GM ADC map (Figure 3n) for 76-space analysis.

\section{Evaluation and Validation}

\subsection{Tissue Classification}

In the absence of digital DTI phantoms, we evaluate tissue classification on ADC and FA images by measuring volume agreement between the segmentation result in DWI/DTI space and that in SPGR space. The average volume agreements for WM, GM and CSF over fifteen cases are $0.88,0.94$ and 0.92 , respectively. These high agreements indicate that the tissue segmentation in DWI/DTI space has good consensus with that in SPGR space. Notably, the volume agreement for GM is the highest, which is mostly desired for GM diffusivity analysis.

\subsection{Heterogeneous Tissue Removal}

A side-effect of the proposed multi-channel fusion is that certain GM regions will be shrunk after the heterogeneous tissue removal. The average volume-shrinking percentage caused by heterogeneous CSF and WM removals over fifteen cases are $15 \%$ and $17 \%$, respectively. This result further confirms that the problem of heterogeneous tissues in co-registration of DWI/DTI and SPGR images is very common. Not surprisely, the heterogeneous CSF tissues could significantly increases the average ADC values. Averaging over the 76 GM structures, the heterogeneous CSF caused an increase of $9 \%$ of the ADC value. For particular GM structures, e.g., the right thalamus in one case, the increased ADC value caused by heterogeneous CSF reaches $29 \%$. After both the heterogeneous CSF and WM removal, the average ADC value slightly increases by $2 \%$. This is expected, as the ADC value of WM is lower than that of GM, and removal of WM would decrease the average ADC in GM.

\subsection{Manual Labeling}

Without digital DTI phantoms, we evaluate our automatic measurements of GM diffusivity by the manual labeling. We randomly selected two normal subjects in our dataset, and two neuroanatomy experts manually painted ten GM ROIs for the two normal brains. The manual tracing was performed on the ADC image directly, and avoidance of including heterogeneous tissues in GM was especially taken care of. In the first case, the averaged differences between computerized results and two manual labeling results over the ten GM ROIs are $1 \%$ and $6 \%$ respectively. As for case 2, the average differences are $6 \%$ and $8 \%$, respectively. Overall, the averaged difference between computerized result and manual labeling is about $5 \%$, which is reasonably low considering the inter-rater difference of $5 \%$. 


\section{Applications}

\subsection{GM Diffusivity Study of Normal Brains}

We applied the 76-space analysis method to fifteen normal brains. The ages of the fifteen normal controls are between 29-51. All of them are male. Figure 4 shows the color-coded GM ADC distribution, where the ADC values of GM structures are mapped onto GM/WM surface of an atlas. The visualization shows that the parietal lobe has higher ADC values, while the temporal lobe has lower ADC values. Also we observed that deep GM structures have much lower ADC values. Figure 4 exhibits that there is no visible difference between the ADC on the right and left hemispheres.
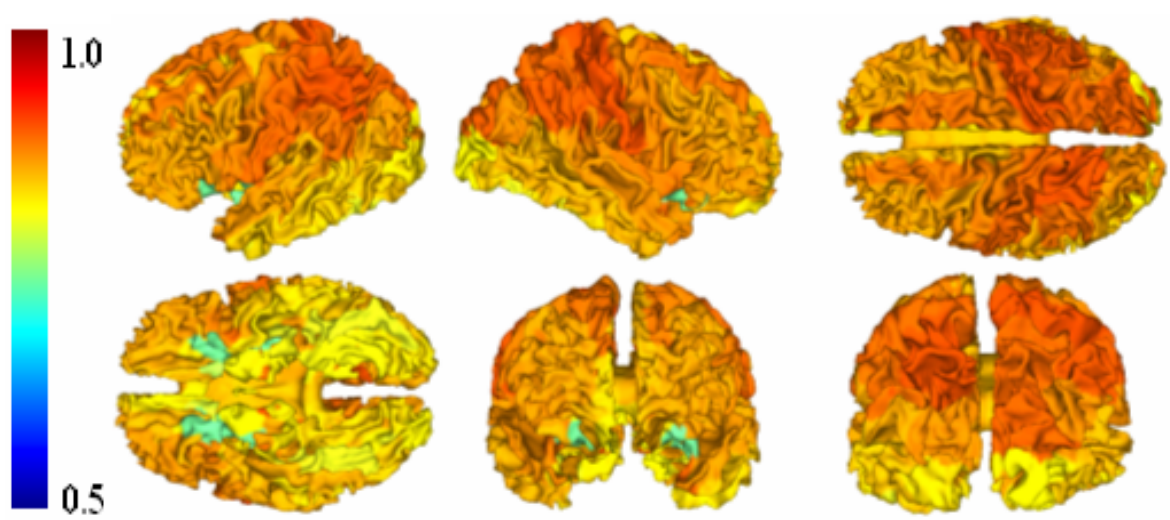

Fig. 4. Color-coded ADC distribution of GM. ADC scale is $10^{-3} \mathrm{~mm}^{2} / \mathrm{sec}$

\subsection{GM Diffusivity Study of Creutzfeldt-Jakob Disease}

We automatically measured the ADC values of the 76 GM structures of 4 CJD patients. We found eight GM structures have significant differences ( $p$-value $<0.05$ ) between CJD and normal brains. Our results show that basal ganglia are frequently involved in these CJD cases, which is in agreement with other research reports [5]. Specifically, the average ADC values of putamen, thalamus, and globus palladus of CJD patients are much lower than those of normal brains. The average ADC values of CJD patients' right and left putamen dropped 29\% and 26\% respectively, compared to those of normal brains. The ADC droppings are confirmed by expert manual tracing.

\subsection{GM Diffusivity Study of Schizophrenia}

We applied the 76-space analysis to study the GM diffusivity of six schizophrenia patients. Their ages are in the range of 28-40. Their genders are all male. Our results show that there are no significant difference ( $\mathrm{p}$-value $>0.05$ ) between ADC values of GM structures of schizophrenia patients and those of normal brains, except that the right and left temporal pole of schizophrenia patients have significant lower ADC values than those of normal brains (p-value: 0.0186 and 0.012). These ADC droppings 
in temporal pole of schizophrenia patients are confirmed by expert manual tracing. In the literature, it is widely reported that temporal pole might be involved in schizophrenia [6]. However, our results need to be verified by future studies involving larger datasets, and its biological meanings are to be further investigated.

\section{Conclusion}

We proposed a new framework of 76-space analysis of GM diffusivity, in which structural information in SPGR image and diffusivity information in DWI/DTI images are integrated through two enabling technologies: high-dimensional hybrid registration and multi-channel fusion. The framework has been applied to study normal brains, CJD and schizophrenia, and produced meaningful results.

\section{Acknowledgements}

We would like to thank Ms. Yi-ru Lin for manual labeling of selected datasets, Dr. Susumu Mori for sharing DTI datasets, Dr. Kabani Noor for sharing brain atlas, and Dr. Martha Shenton (PIs of NIMH R01 MH 50740 and NIH K05 MH 01110) and Dr. Robert McCarley (PIs of NIMH R01 MH 52807 and NIMH R01 MH 40799) for sharing the schizophrenia dataset. This research is funded by a research grant to Dr. Stephen TC Wong by Harvard Center for Neurodegeneration and Repair, Harvard Medical School.

\section{References}

1. M. A. Horsfield, D. K. Jones. Applications of diffusion-weighted and diffusion tensor MRI to white matter diseases - a review. NMR Biomed, 5(7-8):570-7. 2002.

2. P. C. Sundgren, et al, Diffusion tensor imaging of the brain: review of clinical applications, Neuroradiology, 46 (5), 2004.

3. Tianming Liu, D. Shen, C. Davatzikos, Deformable registration of cortical structures via hybrid volumetric and surface warping. NeuroImage, 22 (4) 2004.

4. Y. Zhang et al, Segmentation of brain MR images through a hidden Markov random field model and the expectation-maximization algorithm, IEEE TMI, 20(1), 2001.

5. Geoffrey S. Young, et al, Diffusion Weighted Imaging (DWI) and FLAIR in Creutzfeldt-Jakob disease (CJD): High Sensitivity and Specificity for Diagnosis, in press, American Journal of Neuroradiology.

6. K Kasai, M. E. Shenton, et al. Differences and similarities in insular and temporal pole MRI gray matter volume abnormalities in first-episode schizophrenia and affective psychosis, Arch Gen Psychiatry, 60(11):1069-77, 2003. 